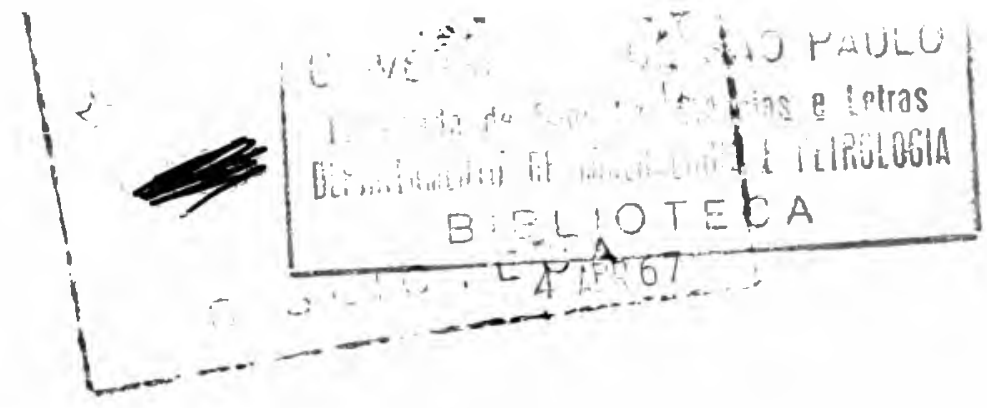

\title{
Mineraes da mina de Furnas Cerussita $^{(1)}$
}

\author{
(1 figura no texto e dois quadros)
}

\author{
R. Saldanha
}

A meio caminho de Apiahy a Iporanga, já neste ultimo municipio, encontra-se a jazida de Furnas, bem conhecida pela exploração da galena argentifera.

Diversos estudiosos voltaram a attenção para os mineraes que occorrem nos veios de Furnas (2) Todos elles assignalaram a existencia de mineraes secundarios sem que, entretanto, nenhum preferisse descrevel-os detidamente. Apenas T. KNECHT (3), em sua

(1) O presente trabalho já foi publicado em avulso e é agora reimpresso, por determinação superior, completado com a parte optica.

(2) Ignacio Ferreira -- Diccionario Geographico das Minas do Brasil, 1885.

Henrique BaUer - As Minas do Yporanga, Revista de Engenharia, n. ${ }^{\circ} 232$, Rio - 1890.

Glycon DE PaIVA - Jazidas de Minerios de Chumbo no Estado de São Paulo. Boletim n. 42 do Serviço Geologico, Rio - 1929.

Luiz Flores de Moraes Rego - Golpe de vista sobre os recursos mineraes de São Paulo, Bcletim da Agricultura, S. Paulo 1930.

Othon Henry Leonardos - Chumbo e Prata no Estado de Sāo Paulo, Boletim n. ${ }^{\circ} 6$ do Departamento Nacional de Producção Mineral - 1934.

THEODCRo KNECHT - As Minas de Funas no Municipio de Iporanga, Boletim de Agricultura, São Paulo - 1929.

Ensaio sobre a occurrencia dos mincraes e minerios do Estado de São Paulo - 1931.

Nota sobre alguns mineraes secundarios de chumbo da mina de Furnas, no Municipio de Iporanga, Boletim de Agricultura, São Paulo - 1932.

(3) Obra citada. 
publicação sobre os mineraes secundarios de Furnas, desenhou alguns crystaes para accentuar o interesse que haveria em uma descripção mais detalhada. Julgamos que o presente trabalho é, assim, uma contribuição interessante ao estudo dos mineraes do Estado de S. Paulo.

O veio de galena, como é notorio, occorre no calcareo da série de S. Roque, apresentando drusas de cerussita, anglesita e calamina.

A cerussita de Furnas é encontrada na parte mais alterada, em que apparece a limonita.

Quando em massas compactas, possue côres variadas, branca, amarella ou preta, por vezes com brilho gorduroso. Os crystaes são incolores, com brilho vitreo e quasi sempre bella transparencia, mas não raro de forte coloração amarella.

Poucas vezes encontram-se crystaes muito desenvolvidos. Os maiores exemplares que observamos medem cerca de $3 \mathrm{~cm}$. na maior dimensão, mas em regra esta não excede 3 a $5 \mathrm{~mm}$.

Apresentam-se, quasi sempre, com estructura radiada em grupamentos irregulares de pequenos individuos dispostos em complicadas rosetas e ligados uns aos outros tão estreitamente que se torna difficil observa-los isolados. Nos pequenos crystaes cuja forma de crescimento permittiu medidas mais perfeitas, ou naquelles que puderam se desenvolver em melhores condições, verificamos, porém, que o habitus da cerussita de Furnas não differe em muito do da cerussita de Lavras (Rio Grande do Sul), estudada por GRODDECK, distinguindo-se sim pela maior riqueza de formas. De facto, GroDDEcK não poude assignalar mais que cinco, $\{010\},\{110\},\{021\}$, $\{041\}$ e $\{111\}$, emquanto nos foi possivel observar cerca de quatro vezes esse numero.

T. KNECHT, sem registrar os valores angulares que teve necessidade de medir, citou algumas formas do material por elle colhido em. Furnas (4) e que não nos foi possivel ter em mãos. De outra parte, tratando-se de formas communs, pudemos verificar a existencia dellas em crystaes da mesma procedencia.

As formas que encontramos são as seguintes:

(4) As fórmas citadas foram $\{001\}\{010\} .\{110\} \quad\{130\} .\{021\}$ e $\{111\}$ ou $\mathrm{OP} \infty \mathrm{P} \propto, \infty \mathrm{P}, \infty \breve{\mathrm{P}} 3,2 \widetilde{\mathrm{P}} \infty$, e $\mathrm{P}$ pela notação de NAUMaNiv, por elle adoptada. 


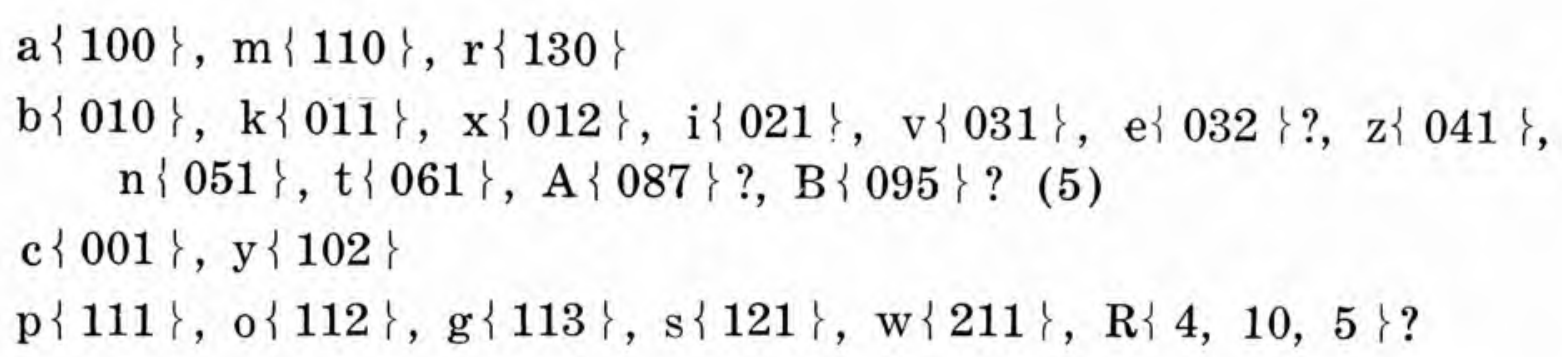

As melhores medidas angulares constam da tabella annexa. Partindo das obtidas para os angulos (110) : (010), (111) : (110)

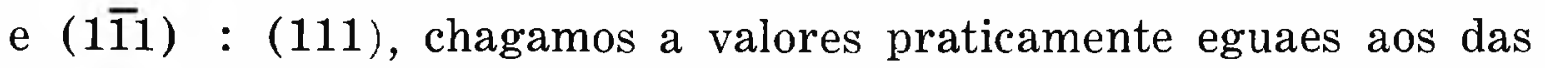
constantes de Kokscharow que são:

$$
a: b: c=0,6100: 1: 0,7230
$$

Servimo-nos destas constantes para calcular os angulos que ficam na sexta columna da tabella.

As combinações sendo variadas verificamos, comtudo, a persistencia das formas $\{\mathrm{okl}\}$ e, em muitas, o desenvolvimento dos prismas verticaes.

Annotamos as combinações encontradas.

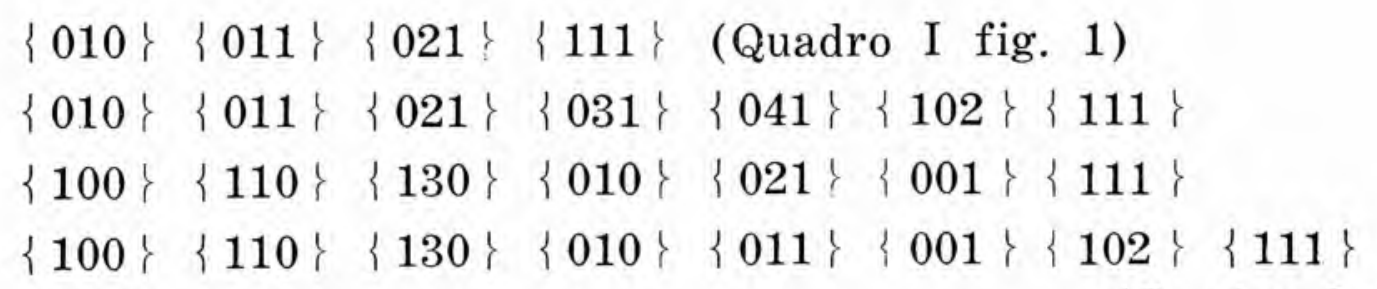

(Quadro I fig. 2)

$\{110\}\{130\}\{010\}\{011\}\{012\}\{021\}\{031\}\{111\}$

$\{110\}\{130\}\{010\}\{011\}\{012\}\{021\}\{031\}\{111\}$

(Quadro I fig. 3)

$\{110\}\{110\} \quad 011\}\{021\}\{031\}\{041\}\{051\}\{061\} \quad\{111\}$ $\{100\}\{110\}\{130\}\{001\}\{102\}\{111\}\{112\}\{113\}\{121\}$

(5) Os prismas $\{087\},\{095\}$ e $\{032\}$ fcram encontrados em um sí excmplar, dando medidas muitn imperfeitas. Apresentamol-os, por isso, sob torma interrogativa. 


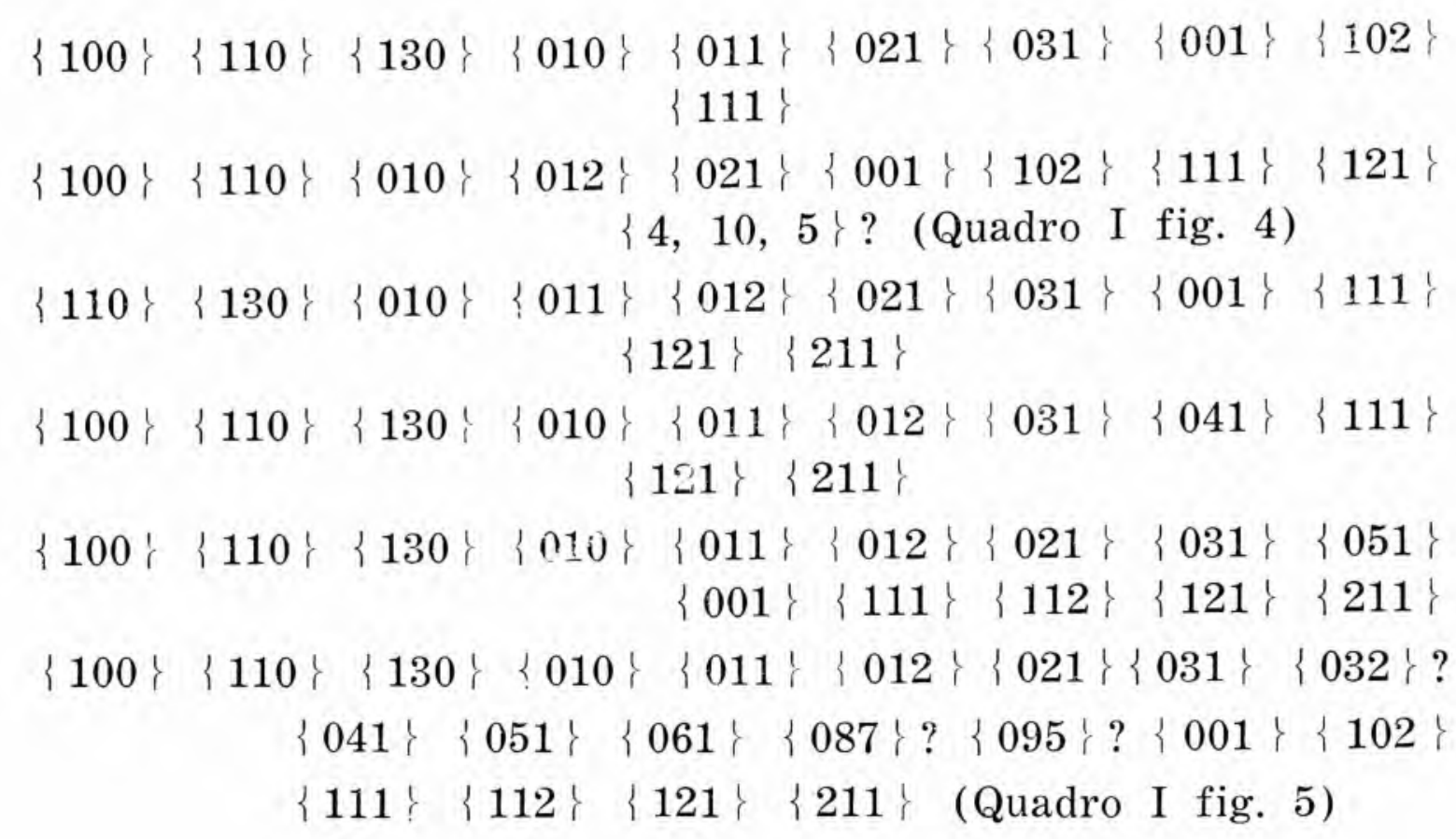

As formas mais frequentes são $\{010\},\{011\},\{021\}$, $\{031\},\{110\},\{130\}$ e $\{111\}$, podendo-se notar que esta ultima foi a unica encontrada, sem excepção, em todos os exemplares observados. Com maior ou menor crescimento, offerece sempre reflexos absolutamente nitidos. As outras pyramides dão tambem bellas facetas, sendo mais commum dentre ellas a forma $\{121\}$

A pyramide $\{4,10,5\}$ encontramol-a em um só crystal (Quadro I fig. 4), bastante estriada, com reflexo multiplo. Damol-a sob a fórma interrogativa porque não são precisas as medidas angulares, tratando-se, ainda, de face que a cerussita de outras localidades não apresenta. Determinamos a sua relação parametrica com o auxilio de angulos medidos aproximativamente e controlamos os que forma com as faces vizinhas, pelo calculo dos mesmos, como damos a seguir:

Angulo

$$
\begin{array}{ll}
(4,10,5) & :(121) \\
(4,10,5) & :(111) \\
(4,10,5) & :(031)
\end{array}
$$

\begin{tabular}{|c|c|}
\hline $5^{\circ} 32^{\prime}$ & $5^{\circ} 44^{\prime}$ \\
\hline $21^{\circ} 59^{\prime}$ & $21^{\circ} 59^{\prime}$ \\
\hline $30^{\circ} 11^{\prime}$ & $29^{\circ} 54^{\prime}$ \\
\hline
\end{tabular}

Valor medido Valor calculado

Os prismas verticaes apresentam-se em faces planas e perfeitas, ora com maior desenvolvimento de $\{110\}$, ora com predominacia de $\{130\}$ 


\begin{tabular}{|c|c|c|c|c|c|c|}
\hline \multirow{2}{*}{\multicolumn{2}{|c|}{$\begin{array}{l}\text { Angulos } \\
\text { Medidos }\end{array}$}} & \multirow{2}{*}{$\begin{array}{c}\text { Numero } \\
\text { de } \\
\text { Medidas }\end{array}$} & \multicolumn{3}{|c|}{ VALORES } & \multirow{2}{*}{$\begin{array}{c}\text { Valores } \\
\text { Calculados }\end{array}$} \\
\hline & & & Maximo & Minimo & Medio & \\
\hline$(110)$ & : $(010)$ & 15 & $58^{\circ} 39^{\prime}$ & $58^{\circ} 36^{\prime}$ & $58^{\circ} 37^{\prime} 12^{\prime \prime}$ & $58^{\circ} 37^{\prime}$ \\
\hline$(130)$ & $:(010)$ & 12 & $28^{\circ} 40^{\prime} 30^{\prime \prime}$ & $28^{\circ} 36^{\prime}$ & $28^{\circ} 39^{\prime} 10^{\prime \prime}$ & $28^{\circ} 39^{\prime} 20^{\prime \prime}$ \\
\hline$(130)$ & : (110) & 17 & $29^{\circ} 59^{\prime} 30^{\prime \prime}$ & $29^{\circ} 55^{\prime}$ & $29^{\circ} 57^{\prime} 10^{\prime \prime}$ & $29^{\circ} 57^{\prime} 40 "$ \\
\hline$(110)$ & $:(1 \overline{10})$ & 7 & $62^{\circ} 48^{\prime}$ & $12^{\circ} 58^{\prime}$ & $62^{\circ} 45^{\prime} 48^{\prime \prime}$ & $62^{\circ} 46^{\prime}$ \\
\hline$(061)$ & $:(010)$ & 2 & $12^{\circ} 58^{\prime}$ & $62^{\circ} 43^{\prime}$ & $12^{\circ} 58^{\prime}$ & $12^{\circ} 59^{\prime}$ \\
\hline$(051)$ & $:(010)$ & 2 & $15^{\circ} 30^{\prime}$ & $15^{\circ} 29^{\prime}$ & $15^{\circ} 29^{\prime} 30^{\prime \prime}$ & $15^{\circ} 27^{\prime} 45^{\prime \prime}$ \\
\hline$(041)$ & $:(010)$ & 7 & $19^{\circ} \quad 7$ & $19^{\circ} \quad 2^{\prime}$ & $19^{\circ} \quad 4^{\prime} 54^{\prime \prime}$ & $19^{\circ} \quad 4^{\prime} 30^{*}$ \\
\hline$(031)$ & $:(010)$ & 12 & $24^{\circ} 46^{\prime} 30^{\prime \prime}$ & $24^{\circ} 42^{\prime}$ & $24^{\circ} 44^{\prime} 50^{\prime \prime}$ & $24^{\circ} 45^{\prime}$ \\
\hline$(021)$ & $:(010)$ & 14 & $34^{\circ} 42^{\prime} 30^{\prime \prime}$ & $34^{\circ} 37^{\prime} 30^{\prime \prime}$ & $34^{\circ} 39^{\prime} 48^{\prime \prime}$ & $34^{\circ} 40^{\prime}$ \\
\hline$(011)$ & $:(012)$ & 8 & $16^{\circ} \quad 30^{*}$ & $15^{\circ} 59^{\prime} 30^{\prime \prime}$ & $16^{\circ}$ & $15^{\circ} 59^{\prime} 30^{*}$ \\
\hline$(012)$ & $:(001)$ & 9 & $19^{\circ} 53^{\prime}$ & $19^{\circ} 50^{\prime} 30^{\prime \prime}$ & $19^{\circ} 51^{\prime} 45^{\prime \prime}$ & $19^{\circ} 52^{\prime} 30^{\prime *}$ \\
\hline (102) & $:(001)$ & 2 & $30^{\circ} 41^{\prime}$ & $30^{\circ} 39^{\prime}$ & $30^{\circ} 40^{\prime}$ & $30^{\circ} 39^{\prime} 12^{*}$ \\
\hline (102) & : (011) & 2 & $45^{\circ} 47^{\prime} 30^{\prime \prime}$ & $45^{\circ} 47^{\prime} 30^{\prime \prime}$ & $45^{\circ} 47^{\prime} 30^{\prime \prime}$ & $45^{\circ} 48^{\prime}$ \\
\hline (111) & $:(010)$ & 3 & $65^{\circ} \quad 3^{\prime}$ & $64^{\circ} 59^{\prime}$ & $65^{\circ} \quad 1^{\prime} 20^{\prime \prime}$ & $65^{\circ} \quad 15^{\circ}$ \\
\hline (111) & : $(110)$ & 13 & $35^{\circ} 47^{\prime}$ & $35^{\circ} 45^{\prime} 30^{\prime \prime}$ & $35^{\circ} 46^{\prime} \quad 5^{\prime \prime}$ & $35^{\circ} 45^{\prime} 48^{*}$ \\
\hline$(111)$ & $:(130)$ & 1 & - & - & $45^{\circ} 22^{\prime}$ & $45^{\circ} 20^{\prime}$ \\
\hline (111) & $:(102)$ & 2 & $31^{\circ} \quad 9^{\prime} 30^{\prime \prime}$ & $31^{\circ} \quad 8^{\prime}$ & $31^{\circ} \quad 8^{\prime} \quad 45^{\prime \prime}$ & $31^{\circ} \quad 8^{\prime}$ \\
\hline (111) & $:(1 \overline{11})$ & 6 & $50^{\circ} \quad 1^{\prime} 30^{*}$ & $49^{\circ} 58^{\prime} 30^{\prime \prime}$ & $49^{\circ} 59^{\prime} 36^{\prime \prime}$ & $49^{\circ} 52^{\prime} 30^{\prime \prime}$ \\
\hline (112) & $:(011)$ & 1 & - & - & $32^{\circ} 53^{\prime} 30^{\prime \prime}$ & $32^{\circ} 53^{\prime} 20^{*}$ \\
\hline (112) & $:(021)$ & 1 & - & - & $44^{\circ} 38^{\prime}$ & $44^{\circ} 38^{\prime} 36^{\prime \prime}$ \\
\hline (112) & : (102) & 6 & $17^{\circ} 17^{\prime}$ & $17^{\circ} 16^{\prime}$ & $17^{\circ} 16^{\prime} 24^{*}$ & $17^{\circ} 16^{\prime} 30^{\prime \prime}$ \\
\hline (112) & : (111) & 7 & $19^{\circ} 29^{\prime}$ & $19^{\circ} 26^{\prime} 30^{\prime \prime}$ & $19^{\circ} 28^{\prime} 20^{\prime \prime}$ & $19^{\circ} 28^{\prime} 12^{\prime \prime}$ \\
\hline (113) & : (112) & 3 & $9^{\circ} 54^{\prime} 30^{\prime \prime}$ & $9^{\circ} 52^{\prime} 30^{\prime \prime}$ & $9^{\circ} 53^{\prime} 30^{\prime \prime}$ & $9^{\circ} 56^{\prime}$ \\
\hline (113) & : (113) & 1 & - & - & $25^{\circ} \quad 3^{\prime} 30^{\prime \prime}$ & $25^{\circ} 16^{\prime}$ \\
\hline (121) & : (010) & 2 & $47^{\circ}$ & $46^{\circ} 58^{\prime}$ & $46^{\circ} 59^{\prime}$ & $47^{\circ}$ \\
\hline$(121)$ & $:(110)$ & 2 & $33^{\circ} 39^{\prime}$ & $33^{\circ} 38^{\prime} 30^{\prime \prime}$ & $33^{\circ} 38^{\prime} 45^{\prime \prime}$ & $33^{\circ} 39^{\prime} 30^{*}$ \\
\hline (121) & : (111) & 10 & $18^{\circ} \quad 1^{\prime}$ & $17^{\circ} 58^{\prime}$ & $18^{\circ} \quad 24^{\prime \prime}$ & $18^{\circ} \quad 15^{\circ}$ \\
\hline$(211)$ & $:(100)$ & 5 & $27^{\circ} 30^{\prime} 30^{\prime \prime}$ & $27^{\circ} 28^{\prime}$ & $27^{\circ} 29^{\prime} 24$ & $27^{\circ} 30^{\prime}$ \\
\hline (211) & : (110) & 2 & $26^{\circ} \quad 5^{\prime}$ & $26^{\circ} \quad 5^{\prime}$ & $26^{\circ} \quad 5^{\prime}$ & $26^{\circ} \quad 5^{\prime}$ \\
\hline (211) & $:(130)$ & 1 & - & - & $48^{\circ} 37^{\prime}$ & $48^{\circ} 29^{\prime} 25^{\prime \prime}$ \\
\hline$(211)$ & : (111) & 7 & $18^{\circ} 40^{\prime} 30^{\prime \prime}$ & $18^{\circ} 38^{\prime} 30^{\prime \prime}$ & $18^{\circ} 39^{\prime} 18^{\prime \prime}$ & $18^{\circ} 39^{\prime}$ \\
\hline (211) & 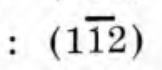 & 1 & - & - & $48^{\circ} 46^{\prime} 30^{\prime \prime}$ & $48^{\circ} 47^{\prime} 10^{\prime}$ \\
\hline$(2 \overline{11})$ & : (130) & 1 & - & 一 & $79^{\circ} 10^{\prime} 30^{\prime \prime}$ & $79^{\circ} 10^{\prime} 12^{\prime \prime}$ \\
\hline$(2 \overline{11})$ & : (211) & 1 & - & - & $31^{\circ} 23^{\prime}$ & $31^{\circ} 23^{\prime} 39^{*}$ \\
\hline
\end{tabular}


O prisma horizontal $\{102\}$, muito menos frequente, dá algumas bôas medidas ao goniometro.

O maior desenvolvimento, porém, é o relativo á zona dos " $x$ ", com o crescimento accentuado do pinacoide $\{010\}$, dando muitas vezes ao crystal o habitus tabular. Esta zona é a que offerece maiores anomalias, sendo communs as estrias e varias perturbações no crescimento das faces. Em $\{010\}$ observa-se, bem pronunciado, o estriamento parallelo a [100]. Nota-se o accrescimento periodico das faces $\{031\}$ e $\{041\}$ em alguns crystaes.

Os pinacoides $\{100\}$ e $\{001\}$ são menos frequentes, encontrando-se o primeiro em pequenas facetas alongadas: attinge o segundo maior desenvolvimento e faz-se notar pelas estrias na mesma direcção das existentes em $\{010\}$; em alguns casos encontramos o pinacoide $\{001\}$ perfeitamente limpido e polido.

Os geminados que estudamos ao goniometro obedecem a uma mesma lei, apresentando como plano de geminação (110). Alguns outros exemplares possuem o habitus em coração, caracteristico da geminação segundo (130), mas não nos foi possivel fazer o controle das medidas angulares, nem mesmo aproximativas.

Apresentando a cerussita habitus diversos e tal seja a justaposição ou entrecruzamento dos crystaes, é possivel observar geminados com os individuos bem distinctos uns dos outros, assim como os que não apresentam angulos reintrantes.

Neste ultimo caso comprehende-se quasi sempre, a combinação de $(010),(1 \overline{1} 0)$ e $(1 \overline{1} 0)$ de dous geminados, com o habitus pseudohexagonal (Quadro II fig. 6). Ás tres faces citadas correspondem respectivamente $(021),(111)$ e $(1 \overline{1} 1)$, assumindo o aspecto de uma pyramide terminal na extremidade livre.

Em outros casos a falsa pyramide é substituida pelo pinacoide (001), bem desenvolvido e com estrias parallelas a [100] nos dous crystaes (Quadro II fig. 7); taes estrias e alguns angulos reintrantes, o das pequenas faces $\mathrm{r}: \overline{\mathrm{r}}, \mathrm{x}: \overline{\mathrm{x}}$ e $\mathrm{s}: \overline{\mathrm{s}}$, demonstram, á mais simples inspecção, a existencia dos geminados.

São frequentes os tri-geminados em cruz, segundo os planos (110) e (110), em que aparece sómente desenvolvida uma metade dos tres individuos como é indicado na fig 8 do quadro II. Quando o habitus tabular é accentuado, os angulos reintrantes tornam-se bem visiveis e os individuos completamente definidos. 
Em differentes exemplares, embora associados da mesma forma, os crystaes offerecem maior desenvolvimento da forma $\{110\}$, accentuado o habitus prismatico e notando-se ainda a bi-terminação em pyramide (Quadro II fig. 9).

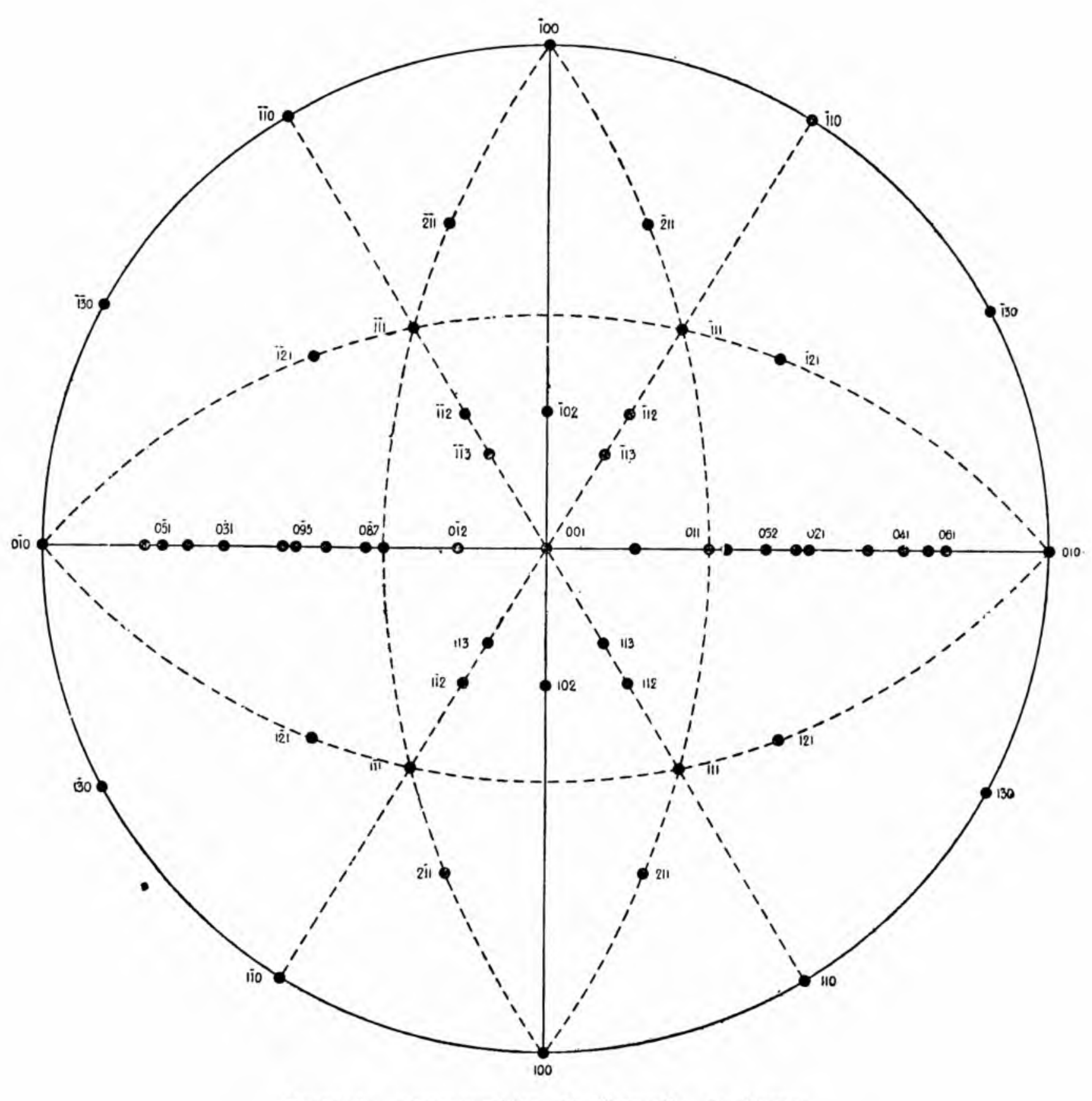

Projecção estereographica da Cerussita de Furnas 
Completando o estudo crystallographico fizemos a medida dos indices de refracção, servindo-nos, respectivamente, dos angulos das faces (130) : (13̄0) util sómente para determinação de $\mathrm{n}_{\alpha \text {, }}$ (102) : (001) para a de $n_{\bar{\beta}}$ e $(021):(0 \overline{1} 0)$ para a de $n_{\gamma}$.

Para a luz de $\mathrm{Na}$ obtivemos os seguintes valores:

$$
\begin{aligned}
& n_{x}=1,7989 \\
& n_{\bar{\gamma}}=2,0722 \\
& n_{\gamma}=2,0741
\end{aligned}
$$

Com a mesma luz procedemos á medida do angulo dos eixos opticos, resultando, para media de cinco determinações, o valor:

$$
2 \mathrm{E}_{\mathrm{a}}=17^{\circ} \quad 21^{\prime}
$$

Conhecendo já o indice medio pudemos calcular o angulo verdadeiro:

\begin{tabular}{|c|c|c|c|c|c|c|}
\hline \multirow{2}{*}{ Autor } & \multirow{2}{*}{$\begin{array}{l}\text { Locali- } \\
\text { dade }\end{array}$} & \multicolumn{3}{|c|}{ Indices } & \multicolumn{2}{|c|}{ Ang. eixos opticos } \\
\hline & & $\mathrm{n}_{\alpha}$ & $n_{\beta}$ & $\mathrm{n}_{\gamma}$ & $\begin{array}{c}2 \mathrm{E}_{\mathrm{a}} \\
\text { (medido) }\end{array}$ & $\frac{2 \mathrm{~V}_{\mathrm{a}}}{\text { (calculado) }}$ \\
\hline H. Ohm (6) & Münster & 1,8033 & 2,0725 & 2.0782 & $17^{\circ} 192 /: 3$ & $8^{\circ} 20^{\prime}$ \\
\hline G. B. Negri (7) & Auronzo & 1,8036 & 2,0765 & 2,0786 & $18^{\circ} 45^{\prime}$ & $9^{\circ} \quad 0^{\prime}$ \\
\hline Schrauf (8) & - & 1,8036 & 2,0763 & 2,0780 & $17^{\circ} \quad 8^{\prime}$ & $8^{\circ} 14^{\prime}$ \\
\hline Des Cloiseaux (9) & - & 1,7980 & 2,0728 & 2,0745 & - & $8^{\circ} 16^{\prime}$ \\
\hline R. Saldanha & Furnas & $\underline{1}, 7989$ & 2,0722 & 2,0741 & $17^{\circ} 21^{\prime}$ & $8^{\circ} 19^{\prime}$ \\
\hline
\end{tabular}

$$
2 \mathrm{~V}_{\mathrm{a}}=8^{\circ} \quad 19^{\prime}
$$

No quadro junto damos os resultados por nós obtidos, comparando-os com os de alguns outros autores.

(6) H. Oнм - N. Jahrb. f. Mineralogie, etc., B. Bd. XIII, 31.

(7) G. B. NegRI - Ref. Zt. für Krystallographie, 19 Bd., 319-320.

(8) Schrauf - Handbuch der Mineralogie, C. Hintze, 1 Bd, 2 Ab, 1H, 3055.

(9) Des Cloiseaux - Idem, idem, idem. 


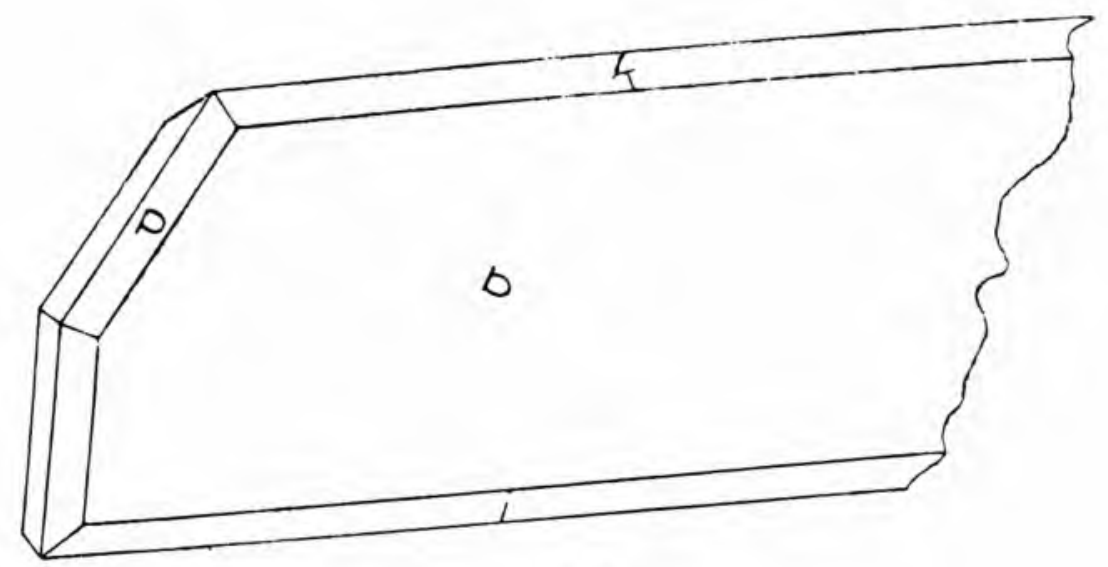

Fig. 1
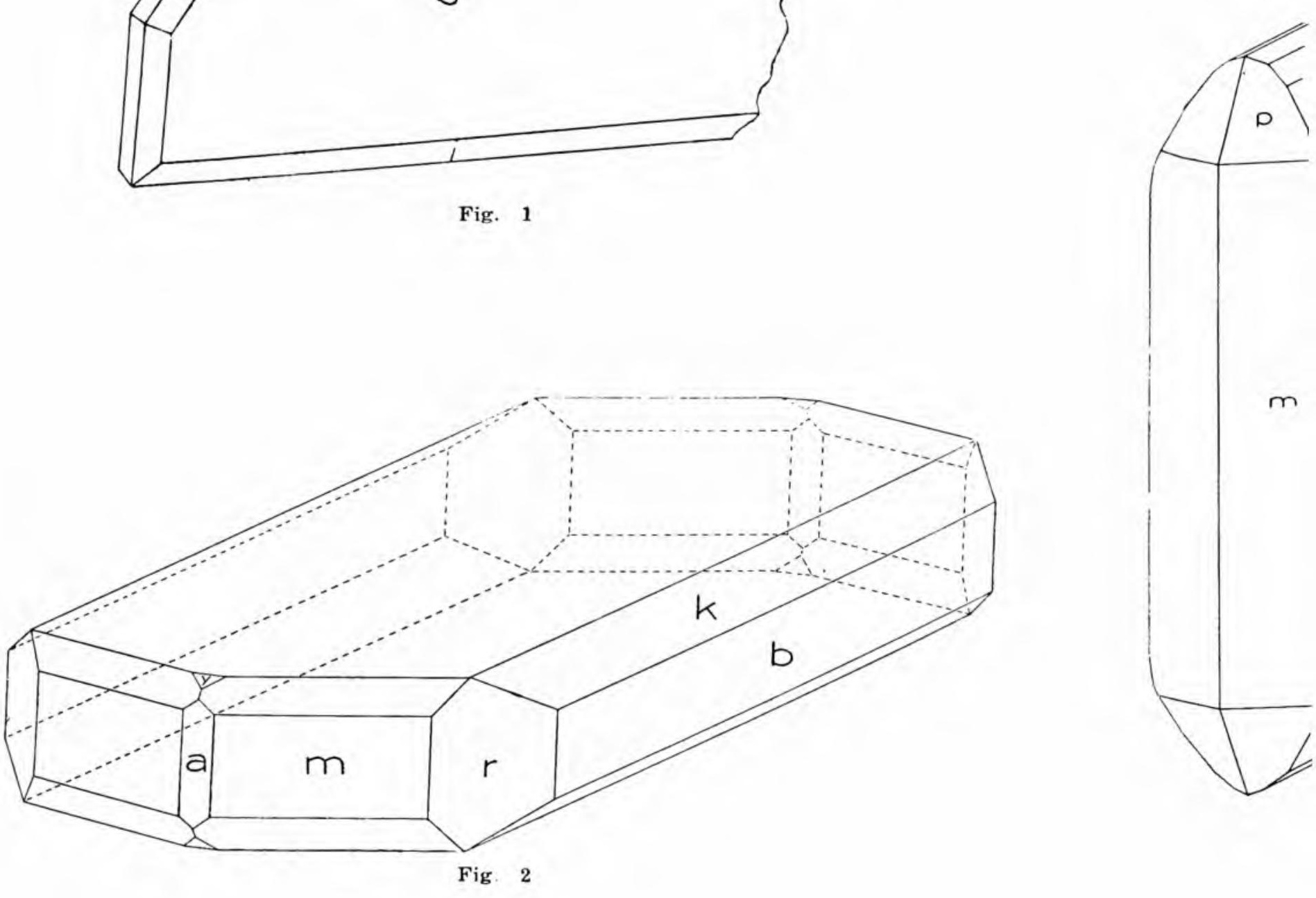



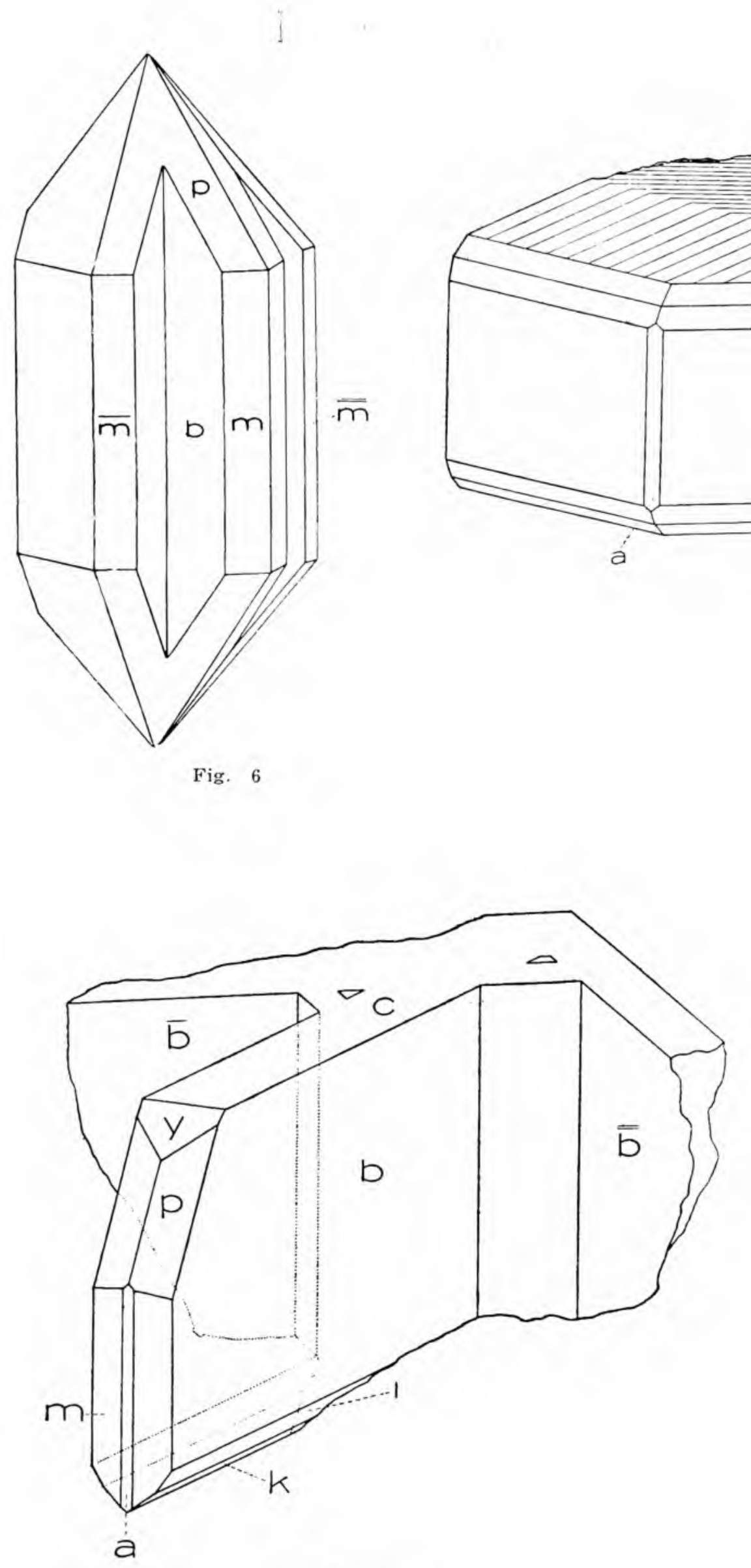

Fig. 8
QUADRO II

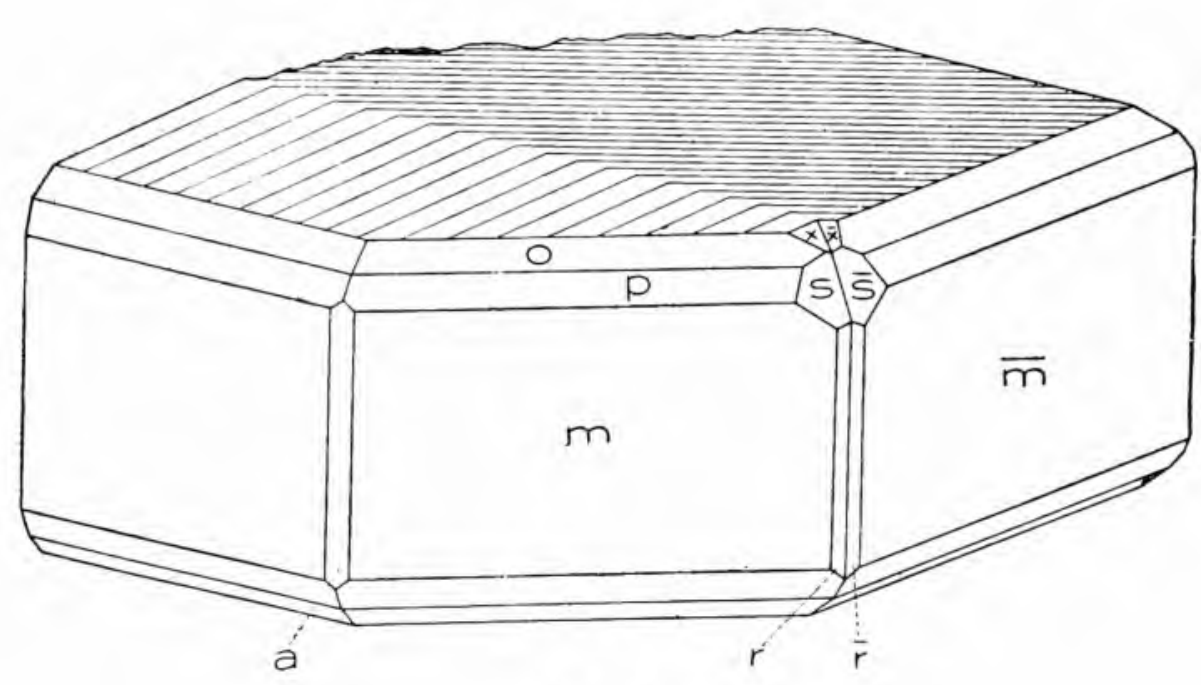

Fig. 7 
SUMMARIUM

\section{CERUSSITA}

Cerussita e Furnas crystallis paucorum amplitudinis centimetrorum, rarissime tria centimetra attingentibus, apparet.

Nullo colore, ubi cristalli forma se ostendit : albo, flavo, vel nigro ubi compacta coalescit.

Quas reperimus formas : $a\{100\}, m\{110\}, r\{130\}, b\{010\}$, $\mathrm{k}\{011\}, \mathrm{x}\{012\}, \mathrm{i}\{021\}, \mathrm{v}\{031\}, \mathrm{e}\{032\}$ ?, $\mathrm{z}\{041\}, \mathrm{n}\{051\}$, t $\{061\} \mathrm{A}\{087\}$ ?, B $\{095\}$ ?, c $\{001\}, \mathrm{y}\{102\}, \mathrm{p}\{111\}, \mathrm{o}\{112\}$, $\mathrm{g}\{113\}, \mathrm{s}\{121\}, \mathrm{w}\{211\}, \mathrm{R}\{4.10 .5\}$ ?. (nova in specie)

Relatio parametrica : a : b : c = 0,6100: $1: 0,7230$

Habitus frequens : tabularis secundum $\{010\}$

Crystalla geminata : secundum $\{110\}$ et $\{130\}$

Refractionis index : $\mathrm{n}_{\alpha}=1,7989 \mathrm{n}_{\hat{\beta}}=2,0722 \mathrm{n}_{\gamma}=2,0741$ Opticorum axium angulus : $2 \mathrm{~T}_{\mathrm{a}}=17^{\circ} 21^{\prime} \quad 2 \mathrm{~V}_{\mathrm{a}}=8^{\circ} 19^{\prime}$ 